\title{
Transforming growth factor $\beta$ induced mutation-associated phenotype in a Chinese family exhibiting lattice corneal dystrophy
}

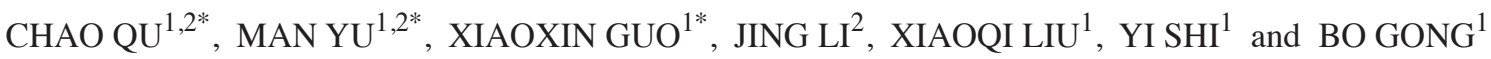 \\ ${ }^{1}$ Sichuan Key Laboratory for Disease Gene Study; ${ }^{2}$ Department of Ophthalmology, \\ Sichuan Academy of Medical Sciences and Sichuan Provincial People's Hospital, School of Medicine, \\ University of Electronic Science and Technology of China, Chengdu, Sichuan 610072, P.R. China
}

Received June 27, 2017; Accepted August 18, 2017

DOI: $10.3892 /$ br.2017.975

\begin{abstract}
Lattice corneal dystrophy type I (LCDI) is associated with a large number of missense mutations in the transforming growth factor $\beta$ induced $(T G F B I)$ gene. The aim of the present study was to analyze TGFBI mutation in a Chinese family with LCDI, and to describe the clinical features and phenotype-genotype correlation within this family. Three generations of this family with LCDI were enrolled in the current study. Complete ophthalmic examinations were performed on all family members and mutation screenings of the coding regions of $T G F B I$ were analyzed using a direct sequencing method. All family members underwent slit-lamp examination, and two patients and one of normal members in the family were evaluated by laser scanning in vivo confocal microscopy. A single heterozygous c.370C $>$ T (p.R124C) mutation was identified in exon 4 of the TGFBI gene in five affected individuals, but not in the other family members and 400 normal control subjects. The affected members exhibited similar clinical features of LCDI, except that patient III:5 presented with mild symptoms. Confocal microscopy in vivo examination demonstrated that the proband (II:2) and his affected niece (III:4) had disruptions in multiple corneal layers, including the basal epithelial cells, stroma cells and Bowman's membrane. Thus, the R124C mutation in the TGFBI gene was identified in a Chinese family with LCDI. These results characterized the clinical features and revealed a genotype-associated phenotype in this family, which may contribute to understanding the pathogenesis of LCDI.
\end{abstract}

Correspondence to: Dr Bo Gong, Sichuan Key Laboratory for Disease Gene Study, Sichuan Academy of Medical Sciences and Sichuan Provincial People's Hospital, School of Medicine, University of Electronic Science and Technology of China, 32 Road West 2, The First Ring, Chengdu, Sichuan 610072, P.R. China E-mail: gongbo2007@hotmail.com

*Contributed equally

Key words: lattice corneal dystrophy type I, transforming growth factor $\beta$ induced, R124C mutation, confocal microscopy

\section{Introduction}

Corneal dystrophy is a group of hereditary diseases characterized by corneal opacities resulting from progressive accumulation of abnormal deposits in corneal stroma, which leads to visual impairment $(1,2)$. Lattice corneal dystrophy (LCD) is one of the most common types of corneal disorderand is characterized by lineal stromal amyloid depositions that usually present bilaterally and symmetrically. Based on age of onset, severity of the phenotype, appearance and depth of the lattice lines, LCD was classified into five subtypes: Type I, IIIA, I/IIIA, IV and IIIB $(3,4)$.

LCD type I (LCDI), the classic form and most frequently encountered type of corneal dystrophy, is an early onset dystrophy exhibiting variable clinical expression $(5,6)$. It is usually inherited as an autosomal dominant, autosomal recessive or X-linked recessive Mandelian inheritance trait. Mutations of the carbohydrate sulfotransferase 6 and transforming growth factor $\beta$ induced (TGFBI) gene have been reported to cause macular corneal dystrophy and corneal disease (4). Currently, LCDI has been associated with a large number of missense mutations with genotype-phenotype correlations in the TGFBI gene, and the International Committee for Classification of Corneal Dystrophies categorized it as LCD-TGFBI type $(3,7)$.

In 1997, the TGFBI gene was first identified as a causative gene for corneal dystrophies by Munier et al (8). This gene, originally termed $\beta$-igh 3 , comprises 17 exons coding for a unique protein of 683 amino acids. So far, $~ 50$ different mutations causing LCD have been identified in the TGFBI gene (www.hgmd.org). The TGFBI mutation spectrum and their clinical consequences have been investigated in patients with LCDI in different ethnic populations (2,7,9-13). For example, the p.Arg124Cys mutation is reported to be associated with the LCDI phenotype in Greek, Japanese, Bangladeshi and Chinese patients (14-19). Therefore, there is a significant correlation between $T G F B I$ mutations and their associated phenotype. However, the exact mechanisms and pathological roles of TGFBI mutations in the development of LCD remain largely unknown, and the correlation between phenotype and genotype of TGFBI in the Chinese population has not been extensively investigated.

In the present study, a Chinese family with LCDI was investigated to determine the mutation underlying this disease, and 
to characterize the clinical features and phenotype-genotype correlation of this family.

\section{Materials and methods}

Study participants. The current study was approved by the Institutional Review Boards of Sichuan Academy of Medical Sciences and Sichuan Provincial People's Hospital, University of Electronic Science and Technology of China (Chengdu, China). Written informed consent was obtained from all subjects prior to the study. All procedures in the present study adhered to the tenets of the Declaration of Helsinki. Five affected and 11 unaffected individuals from a Chinese family (Fig. 1A) were enrolled in the study after obtaining informed consent. Slit-lamp examination was performed for all participating individuals in this family to determine whether they were affected or unaffected with corneal dystrophies and to determine the disease phenotype. Three individuals in the family (II:2, III:1 and III:4) were evaluated by laser scanning in vivo confocal microscopy (Heidelberg Retina Tomograph II, Rostock Corneal Module; Heidelberg Engineering $\mathrm{GmbH}$, Heidelberg, Germany). Detailed clinical histories of all the participating subjects, such as the age of onset, initial signs and symptoms, progression of disease, other ocular therapeutic procedures, and so on, were obtained. A total of 400 unrelated healthy control subjects were recruited from the Hospital of University of Electronic Science and Technology of China. These control subjects had no medical history of associated visual disorders.

DNA extraction. All genomic DNA was extracted from $2 \mathrm{ml}$ peripheral blood, using a blood DNA extraction kit (QIAamp DNA Blood Midi kit, cat. no. 51106; Qiagen GmbH, Hilden, Germany) according to the manufacturer's protocol. DNA samples were stored at $-20^{\circ} \mathrm{C}$ until used. DNA integrity was evaluated by $1 \%$ agarose gel electrophoresis. Briefly, $5 \mu \mathrm{l}$ of DNA with $1 \mu$ l loading buffer were loaded into $1 \%$ agarose gel and run at $120 \mathrm{~V}$ for $30 \mathrm{~min}$ in an electrophoresis apparatus (Bio-Rad Laboratories, Inc., Hercules, CA, USA).

Mutation screening. The coding sequences of TGFBI (NM_000358.2) was amplified by polymerase chain reaction (PCR) with 35 cycles $\left(30 \mathrm{sec}\right.$ at $95^{\circ} \mathrm{C}$ for initial denaturation, $30 \mathrm{sec}$ for annealing at different temperatures and $30 \mathrm{sec}$ at $72^{\circ} \mathrm{C}$ for extension), using a MyCycler thermocycler (Bio-Rad Laboratories, Inc.). Sequencing primers forthe flanking sequence of each exon were designedusing Primer 5.0 software (Premier Biosoft International, Palo Alto, CA, USA). Amplified PCR products were purified with spin columns (QIAquick PCR Purification kit, cat. no. 28104; Qiagen, Inc., Valencia, CA, USA) and sequenced directly (BigDye Terminators Sequencing kit (cat. no. 4337455; Applied Biosystems; Thermo Fisher Scientific, Inc., Waltham, MA, USA) in two directions using an automated genetic analysis system (model 3130; Applied Biosystems; Thermo Fisher Scientific, Inc.). Multiple sequence alignment ofthe human TGFBI protein was performed along with other TGFBI proteins across different species to assessfor the conservation of residues. The possible damaging effects of this mutation on the structure and function of TGFBI were predicted using
A

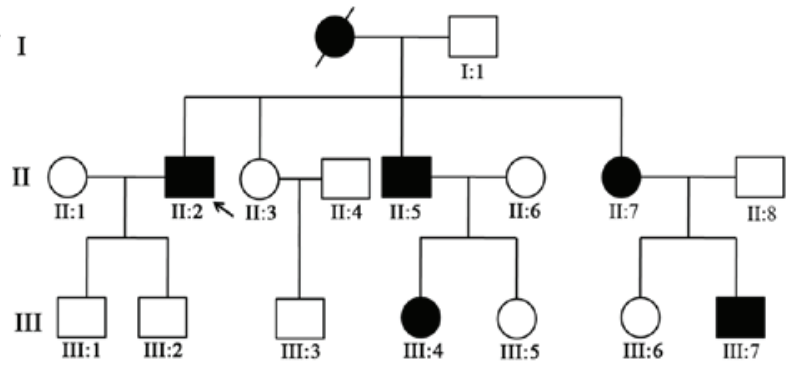

B $\quad \frac{C T G}{L} \frac{T A C A C G}{Y} \frac{G A C}{D} \frac{C G C}{R} \frac{A C G}{T} \frac{G A G}{E} \frac{A A G}{K} \frac{C T G}{L}$

Wild-type

c. $370 \mathrm{C}<\mathrm{T}$

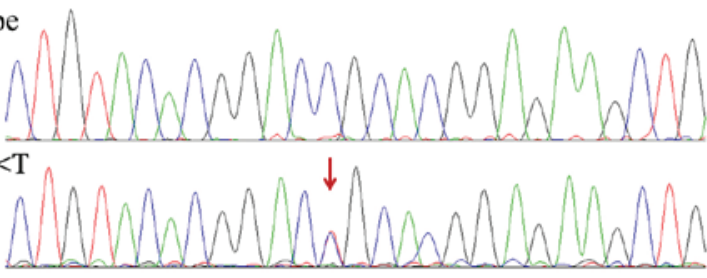

C

\begin{tabular}{lll} 
& \multicolumn{2}{c}{ p.Arg124Cys } \\
H.sapiens & TLGVVGSTTTQLYTD & TEKLRPEMEGPGS---FTIF \\
P.troglodytes & TLGVVGSTTTQLYTD & TEKLRPEMEGPGS---FTIF \\
M.mulatta & TLGVGGSTTQLYTD & TEKLRPEMEGPGS---FTIF \\
C.lupus & TLGVVGSTTTLYTD & TEKLRPEMEGPGS---FTIF \\
B.taurus & TLGVGGTTTQLYTD & TEKLRPEMEGPGS---FTIF \\
M.musculus & TMGVVGTTTQLYTD & TEKLRPEMEGPGS---FTIF \\
R.norvegicus & TLGIVGSTTTQLYTD & TEKLRPEMEGPGS---FTIF \\
G.gallus & TLGVVGSATTQLYSD & SN-LRPEIEGPGT---FTIF \\
D.rerio & TLSVVEATTTKMYSE & AK-LQEEIEGPGS---FTFF \\
D.melanogaster & TASDMGAKQF-LESA & TAGELADMLGAGSGKKVTLF \\
X.tropicalis & TLGVVGAATTQLYSD & AN-LRPEIEGPGS---FTIF
\end{tabular}

Figure 1. R124C mutation in the TGFBI gene was identified in a Chinese family with LCDI. (A) Heredity of the family with LCDI. Solid symbols indicate affected individuals, open symbols indicate unaffected individuals and arrows indicate the proband. (B) Representative chromatogram of the R124C mutation in TGFBI. (C) Orthologous protein sequence alignment of TGFBI from different species, the mutated residue demonstrating conservation of arginine(R) at codon 124 was shaded in red. TGFBI, transforming growth factor $\beta$ induced; LCDI, lattice corneal dystrophy type I.

Sorting Intolerant From Tolerant (SIFT; http://sift.jcvi.org) and PolyPhen-2 (http://genetics.bwh.harvard.edu/pph2/).

\section{Results}

Clinical data. A family including 16 members from the Sichuan province of China was recruited in the current study (Fig. 1A). Ophthalmic examinations identified five affected individuals (II:2, II:5, II:7, III:4 and III:5) among the family members. The affected subjects with LCDI exhibited similar clinical features, except that patient III:5 presented with mild symptom. The proband, patient II: 2 in this family, was initially diagnosed as LCDI in each eye when he visited the Hospital of University of Electronic Science and Technology of China at the age of 43 years. The patient had experienced bilateral foreign body sensation and visual defects since the age of 26 , and progressive deterioration in visual acuity bilateral over the past 10 years (Table I). Slit-lamp examination demonstrated various distinct linear deposits in the superficial stroma of the central corneal accompanied by epithelial erosions (Fig. 2). The brother and sister of the proband (II:5 and II:7) were 
Table I. Clinical data of family members with LCDI.

\begin{tabular}{lcccc}
\hline Patient & Sex/age & $\begin{array}{c}\text { Age } \\
\text { at onset } \\
\text { (years) }\end{array}$ & $\begin{array}{c}\text { Duration } \\
\text { (years) }\end{array}$ & $\begin{array}{c}\text { Surgical treatment } \\
\text { (patient's age } \\
\text { when performed) }\end{array}$ \\
\hline II:2 & Male/43 & 26 & 20 & NK \\
II:5 & Male/41 & 24 & 18 & PKP (37-year-old) \\
II:7 & Female/38 & 23 & 16 & PKP (35-year-old) \\
III:4 & Female/19 & 19 & 1 & NK \\
III:5 & Male/15 & 24 & 5 & NK \\
\hline
\end{tabular}

LCDI, lattice corneal dystrophy type I; NK, no keratoplasty; PKP, penetrating keratoplasty.
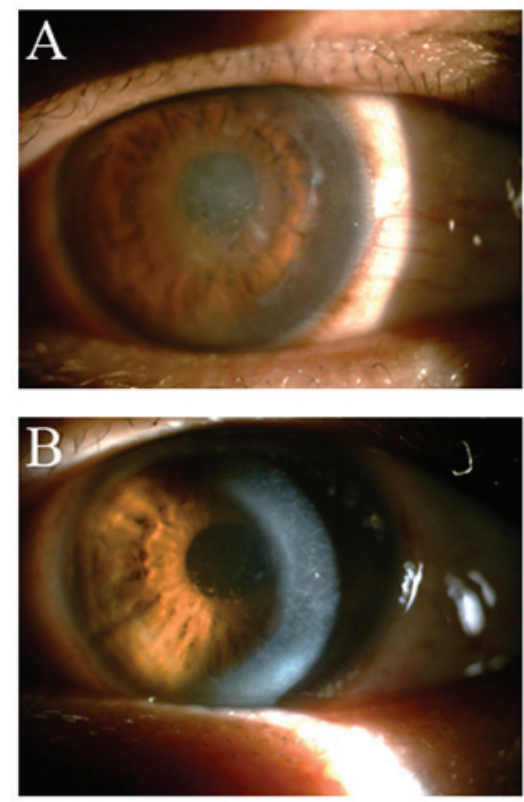

Figure 2. Phenotype of the proband. Slit-lamp examination of the cornea in the same patient showing (A) linear deposits and (B) corneal epithelial erosions in the stroma.

also found to be affected with LCDI. They began to exhibit the initial symptom with a mild foreign body sensation at approximately $21-25$ years old and exhibited obvious visual impairment.

The nieces of the proband (III:4 and III:5) were 19 and 15 years old, respectively. They complained of bilateral blurred vision, recurring ocular pain, photophobia and tearing. The two patients had multiple linear deposits and a few confluent opacities in the anterior stroma of the central cornea. An obvious visual defect was detected in III:4, but not in III:5.

Mutation screening of TGFBI in the family with LCD. Sequencing analysis of the TGFBI gene revealed a heterozygous mutation, c.370C $>\mathrm{T}$ (p.R124C), which was located at nucleotide 370 in the coding sequence of exon 4 (Fig. 1B). This missense mutation was present in the five affected patients (II:2, II:5, II:7, III:4 and III:5). This mutation was not observed in the unaffected family members and 400 normal control subjects. Comparative amino acid sequence alignment

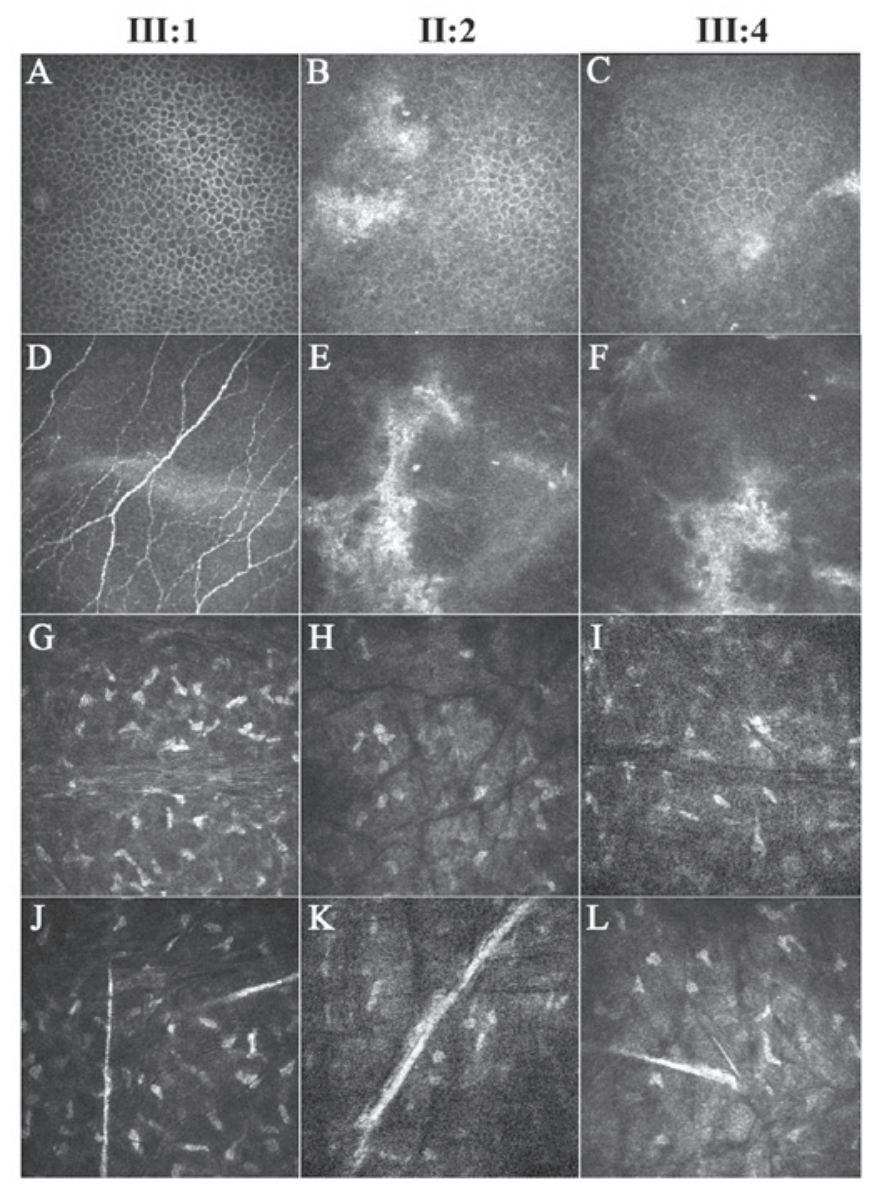

Figure 3. Laser scanning in vivo confocal microscopy findings of the proband (II:2), his affected niece (III:4) and one of the healthy individuals (III:1) (A-C) The basal epithelial cells exhibited more irregular hyperreflective dots in the patients, when compared with the healthy individuals. (D-F) In the lamina elastic anterior (Bowman's membrane), focal depositions of reflective materials, and diffuse and decreased nerve fibers were observed in the patients. (G-I) In the superficial one-third of the stroma, the number of cell nuclei was largely reduced, and collagenous fibers were thinner and fewer in patients than in the healthy individuals. (J-L) In the deeper one-third of the stroma, coarser nerve fibers across the layer were detected in the patients. The frame size for each figure is $400 \times 400 \mu \mathrm{m}$.

of other TGFBI proteins across different species revealed that the mutation occurred at highly conserved positions (Fig. 1C). SIFT and PolyPhen-2 predicted this mutation to be damaging, and it may result in a substitution from arginine to cysteine at position 124.

Confocal microscopy examination. In order to determine whether the detected mutation in the family is associated with LCDI, in vivo confocal microscopy was performed to investigate the difference in corneal structure between healthy and affected members. Laser scanning in vivo confocal microscopy was performed on the proband (II:2), his affected niece (III:4) and one of the healthy individuals (III:1) in the family. The basal epithelial cells of patients were characterized by irregular hyperreflective dots, compared with that of healthy individuals (Fig. 3A-C). In the lamina elastic anterior (Bowman's membrane), numerous nerve fibers were clearly detected in the healthy individual, whereas, focal depositions of reflective materials and decreased nerve fibers were identified in the patients (Fig. 3D-F). In the superficial one-third of 
the stroma, the number of cell nuclei was largely reduced, and collagenous fibers were thinner and fewer in the patients than in the healthy individuals (Fig. 3G-I). In the deeper one-third of the stroma, there were coarser nerve fibers across the layer detected in the patients compared with those in the healthy individuals (Fig. 3J-L). The two patients and the healthy individual exhibited a normal posterior elastic lamina (Descemet's membrane) and endothelium cells, except that the endothelial cells in the patients were visualized faintly, although they appeared to be normal (data not shown).

\section{Discussion}

LCDI is an autosomal, dominantly inherited corneal amyloidosis associated with severe visual defects, and mutations in the TGFBI gene are the main causes of LCDI (7). In the present study, a heterozygous mutation, c.370C $>$ T (p.R124C) was detected in TGFBI and the phenotypic characteristics in a Chinese family affected with LCDI were described. TGFBI mutations have been reported to be closely involved with at least five types of inherited corneal dystrophy. Among those mutations reported, R124 in TGFBI appeared to be a 'hot-spot' point mutation detected in different types of corneal dystrophy in various ethnic groups worldwide (7). R124 in exon 4 of $T G F B I$ is conserved among numerous different species (Fig. 1C), indicating that this residue is an important functional and structural site of the protein. Furthermore, it is important to investigate the mutation underlying this disease and to characterize the clinical features and phenotype-genotype correlations within this family.

In the present study, five affected individuals among the 16 family members were diagnosed with LCDI, although there is already a study of phenotypic variability with the R124C mutation in LCDI pedigrees (17). LCD is generally divided into three subtypes and is classified predominantly by clinical and pathological findings. The underlying mechanism of the phenotypic variability for corneal dystrophy with the same mutation and even within the same family requires further investigation. Therefore, it is necessary to identify the specific mutation in patients to assess a final diagnosis due to genetic heterogeneity. In the current family, the affected members with the R124C mutation exhibited similar clinical features of LCDI. They all had prominent delicate linear opacities that tended to predominantly be in the superficial corneal stroma accompanied with epithelial erosions, although patient III:5 exhibited mild symptoms. Molecular genetic analysis, which is important in establishing the classification of corneal dystrophy, may reveal reliable clinical diagnostic criteria and may improve the accuracy of the clinical diagnosis. The patient, III:5, who carried the same R124C mutation as the other four patients, was thus diagnosed as LCDI although she did not exhibit typical clinical features of LCDI. It is also possible that she was younger and may develop corneal dystrophy in the future, indicating that genetic analysis may be used during prenatal and postnatal DNA diagnosis for clinical work.

In order to confirm the clinical features of patient III:4, she was recruited for examination by in vivo confocal microscopy. The images indicated that multiple corneal layers, including the basal epithelial cells, stroma cells and Bowman's membrane, were affected in the two patients (the proband,
II:2 and III:4) of this family (Fig. 3). The proband seemed to exhibit stronger irregular hyperreflective materials compared with patient III:4 in the basal epithelial cells and Bowman's membrane (Fig. 3A-C). It is likely that patient III:4 was markedly younger than the proband. Patient III:4 was found to have milder symptom than the proband, indicating that the worse the visual acuity is, the more likely that the morphology of corneal layers was affected. During confocal microscopy and slit-lamp examination of the affected members, their corneal epithelia were particularly fragile and corneal epithelial erosions were identified. They also developed photophobia and lacrimation by microscopic examination. Observations of the abnormal morphology of Bowman's membrane in the patients (Fig. 3D-F) identified that the density of nerve fibers was significantly decreased and even disappeared; it is likely that the damaged Bowman's membrane cannot provide normal nutrition for corneal nerve growth, thus leading to corneal deposits. If this corneal dystrophy was due to lack of nutrition, the disease maybe treated with neurotrophic factors. The TGFBI protein is presumably involved in cell adhesion and migration of various cell types, including epithelial cells, fibroblasts, endothelial cells, and vascular smooth muscle cells (20). Therefore, patients with the TGFBI R124C mutation may exhibit relaxation of the connection between the corneal epithelial cells and the entire epithelial layer, leading to the abnormity of Bowman stromal corneal dystrophy in this family. Furthermore, nerve fibers may not grow into the Bowman's layer and the corneal epithelial cells lack nerve nutrition, resulting in exfoliation of the corneal epithelium (Fig. 2).

In conclusion, a mutation in the TGFBI gene was identified in a Chinese family with LCDI. The current results characterized the clinical features of LCDI and revealed a clear genotype-associated phenotype in this family, which may contribute to further understanding the role of this TGFBI mutation in the development of LCDI. However, the composition of the affected corneal layer in patients remains unknown and thus its specific underlying mechanism requires further investigation. Future functional work is required to confirm the role of $T G F B I$ and the underlying mechanisms in the disease.

\section{Acknowledgements}

The authors would like to thank the family and the healthy volunteers for their participation in the study. The study was supported by grants from the Natural Science Foundation of China (grant nos. 81371048, 81570848, 81170882 and 81570888) and the Department of Science and Technology of Sichuan Province (grant nos. 2015JZ0004, 2015HH0031 and 2014JZ0004).

\section{References}

1. Klintworth GK: The molecular genetics of the corneal dystrophies - current status. Front Biosci 8: 687-713, 2003.

2. Sacchetti M, Macchi I, Tiezzi A, La Cava M, Massaro-Giordano G and Lambiase A: Pathophysiology of corneal dystrophies: From cellular genetic alteration to clinical findings. J Cell Physiol 231: 261-269, 2016.

3. Weiss JS, Møller HU, Lisch W, Kinoshita S, Aldave AJ, Belin MW, Kivelä T, Busin M, Munier FL, Seitz B, et al: The IC3D classification of the corneal dystrophies. Cornea 27 (Suppl 2): S1-S83, 2008. 
4. Schorderet D: Corneal dystrophies: Overview and summary. Prog Mol Biol Transl Sci 134: 73-78, 2015.

5. Klintworth GK: Advances in the molecular genetics of corneal dystrophies. Am J Ophthalmol 128: 747-754, 1999.

6. Lisch W and Seitz B: Lattice corneal dystrophy type 1: An epithelial or stromal entity? Cornea 33: 1109-1112, 2014.

7. Lakshminarayanan R, Chaurasia SS, Anandalakshmi V, Chai SM, Murugan E, Vithana EN, Beuerman RW and Mehta JS: Clinical and genetic aspects of the TGFBI-associated corneal dystrophies. Ocul Surf 12: 234-251, 2014.

8. Munier FL, Frueh BE, Othenin-Girard P, Uffer S, Cousin P, Wang MX, Héon E, Black GC, Blasi MA, Balestrazzi E, et al BIGH3 mutation spectrum in corneal dystrophies. Invest Ophthalmol Vis Sci 43: 949-954, 2002.

9. Dudakova L, Palos M, Jirsova K, Skalicka P, Dundr P and Liskova P: Novel TGFBI mutation p.(Leu558Arg) in a lattice corneal dystrophy patient. Ophthalmic Genet 37: 473-474, 2016.

10. Chae H, Kim M, Kim Y, Kim J, Kwon A, Choi H, Park J, Jang W, Lee YS, Park SH and Kim MS: Mutational spectrum of Korean patients with corneal dystrophy. Clin Genet 89: 678-689, 2016.

11. Cai J, Zhu L, Zha Y and Kang Q: TGFBI gene mutation analysis in Chinese families with corneal dystrophies. Genet Test Mol Biomarkers 20: 388-392, 2016.

12. Ann LB, Abbouda A, Frausto RF, Huseynli S, Gupta K, Alió JL and Aldave AJ: Variant lattice corneal dystrophy associated with compound heterozygous mutations in the TGFBI gene. Br J Ophthalmol 101: 509-513, 2017.

13. Costagliola C, Romano V, Cifariello F, Aceto F and Porcellini A: Lattice corneal dystrophy: A report of two cases in twin sisters due to 3 mutations (T1620C, C1416T, A1924G) in the TGFBI (BIGH3) gene. Clin Ter 165: e73-e75, 2014.
14. Hellenbroich Y, Tzivras G, Neppert B, Schwinger E and Zühlke C: R124C mutation of the betaIGH3 gene leads to remarkable phenotypic variability in a Greek four-generation family with lattice corneal dystrophy type 1. Ophthalmologica 215: 444-447, 2001.

15. Yoshida S, Yoshida A, Nakao S, Emori A, Nakamura T, Fujisawa K, Kumano Y and Ishibashi T: Lattice corneal dystrophy type I without typical lattice lines: Role of mutational analysis. Am J Ophthalmol 137: 586-588, 2004.

16. El-Ashry MF, Abd El-Aziz MM, Ficker LA, Hardcastle AJ, Bhattacharya SS and Ebenezer ND: BIGH3 mutation in a Bangladeshi family with a variable phenotype of LCDI. Eye (Lond) 18: 723-728, 2004

17. Liu Z, Wang YQ, Gong QH and Xie LX: An R124C mutation in TGFBI caused lattice corneal dystrophy type I with a variable phenotype in three Chinese families. Mol Vis 14: 1234-1239, 2008.

18. Courtney DG, Poulsen ET, Kennedy S, Moore JE, Atkinson SD, Maurizi E, Nesbit MA, Moore CB and Enghild JJ: Protein composition of TGFBI-R124C- and TGFBI-R555W-associated aggregates suggests multiple mechanisms leading to lattice and granular corneal dystrophy. Invest Ophthalmol Vis Sci 56: 4653-4661, 2015

19. Yang QN, Zhao YW, Guo LH, Yan NH, Liu XY and Cai SP: Arg124Cys mutation of the TGFBI gene in a Chinese pedigree of Reis-Bücklers corneal dystrophy. Int J Ophthalmol 4: 235-238, 2011.

20. Runager K, Enghild JJ and Klintworth GK: Focus on molecules: Transforming growth factor beta induced protein (TGFBIp). Exp Eye Res 87: 298-299, 2008. 